\title{
STIMULANT DRUG EFFECTS ON ATTENTION DEFICIT/HYPERACTIVITY DISORDER: A REVIEW OF THE EFFECTS OF AGE AND SEX OF PATIENTS.
}

\author{
Christine Cornforth ${ }^{1}$, Edmund Sonuga-Barke ${ }^{1,2^{*}}$, David Coghill ${ }^{3}$
}

1. Institute for Disorders of Impulse and Attention, School of Psychology, University of Southampton.

2. Department of Experimental Clinical \& Health Psychology, Ghent University.

3. Department of Psychiatry, University of Dundee.

* Correspondence - Professor Edmund Sonuga-Barke. Institute for Disorders of Impulse \& Attention, School of Psychology, University of Southampton, Southampton SO17 1BJ, UK. Tel: +44 (0)23 8059 4604. Fax: +44 (0)23 8059 4597. e-mail: ejb3@ soton.ac.uk. 


\section{ABSTRACT}

Objective: Because dopamine functioning varies by sex and age it might be expected that the effects of methylphenidate or amfetamine, the psychostimulants used for the treatment of Attention Deficit /Hyperactivity Disorder (ADHD), will also be moderated by these factors. Here we review the published literature on whether stimulant effects in ADHD symptoms vary by age and sex.

Method: We searched for studies published between 1989 until October 2009. Databases searched included U.S. National Library of Medicine (PubMed), Medline, EMBASE, PsycINFO and ISI Web of Knowledge. Firstly, we reviewed the effects of stimulant drugs on male and female patients and patients of pre-school, middle childhood, adolescence and adulthood. Secondly, we reviewed studies that directly tested the moderating effect of age and sex.

Results: Randomised controlled trials confirm that stimulant medication is efficacious for, and well tolerated by, males and females and patients across the age range; although preschoolers appear to have a less beneficial response and more side effects. Few studies that specifically examined the moderating effect of age and/or sex were identified. For sex, no effects on overall response were found, although one study reported that sex moderated methylphenidate pharmacodynamics. The few effects found for age were small and inconsistent.

Conclusions: The available evidence suggests that stimulant medication, when appropriately administered, has efficacy as an ADHD treatment for both sexes and across all ages. There are currently too few published papers examining the effects of sex and age to draw strong conclusions about moderation. Further studies of the pharmacodynamics of stimulants on 
symptoms measured using objective tests in the laboratory or classroom setting need to be undertaken.

Key words: methylphenidate, amfetamine, psychostimulants, treatment response; sex; gender, age; attention deficit hyperactivity disorder; ADHD; psychopathology; medication; moderation; prediction. 


\section{INTRODUCTION}

Background: Attention Deficit/Hyperactivity Disorder (ADHD) is a chronic and impairing disorder, marked by a pervasive and persistent pattern of inattention, overactivity and impulsiveness; affecting around five percent of the population of children and adolescents. It is associated with significant costs to patients, families and society, as well as burden to social and health care services [1]. Over the long term, untreated ADHD is associated with school failure, delinquency and substance abuse [2-5].

Stimulant and non-stimulant treatments for ADHD: A combination of pharmacological and psychological therapies are recommended for the treatment of ADHD [6, 7]; and a number of different medications (stimulants and non-stimulants) which are licensed in the US and Europe have been shown to have both efficacy and tolerability. This review of sex and age effects on treatment response will focus on stimulant medications.

Stimulant medications are considered a first line of treatment for ADHD [6, 7] leading to a clinically significant reduction of symptoms in around 70 percent of cases [8-10]. In addition, they have also shown some efficacy in the treatment of other conditions, such as narcolepsy and resistant depression $[11,12]$. There are two main classes of stimulants used to treat ADHD; methylphenidate (MPH) and amfetamine (AMF).

MPH is available in several formulations, with different delivery mechanisms that result in varying pharmacokinetic $(\mathrm{PK})$ profiles and different patterns of symptom control across the day [13]. Immediate release (IR) MPH, which has been available for over 50 years and was the mainstay of ADHD treatment for a long time, is associated with a rapid onset of action (around 30 minutes post dose) and relatively rapid offset [around 4 hours post dose; 14]. Consequently, IR MPH needs to be taken several times a day for adequate symptom cover. Swanson and colleagues [summarised in, 13] investigated the potential for developing an 
effective 'once a day extended release (ER) preparation', and as a result of this there are now various oral ER preparations, several of which are licensed in European countries (e.g. Concerta XL, Equasym XL, Medikinet XL and Ritalin LA). These preparations use various technologies to deliver an initial IR bolus followed by an ER dose designed to last for either 8 or 12 hours post dose. There is also, in the US but not Europe, a MPH transdermal patch technology that facilitates a more flexible ER delivery [15]. The relationship between the PK and pharmacodynamics (PD) of MPH is complex and incompletely understood; however recent studies have suggested that the main clinical effects closely follow the predicted PK profiles $[16,17]$.

The precise pharmacological mechanism of action of MPH is currently unknown, with some of the most fundamental issues being debated. It is understood that MPH results in increased levels of extra-cellular dopamine (DA) by blocking DA transporters (DAT) [18]. Grace (2001) has proposed that these increased levels of tonic extrasynaptic DA may stimulate impulse regulating DA autoreceptors resulting in a decrease in phasic DA release and phasic DA levels [19]. On the other hand, Volkow and colleagues (2001) have proposed that the blocked DAT overcomes the inhibitory effects on the autoreceptors, resulting in a net accumulation of DA in the synapse and amplification of DA signals from both tonic and phasic DA cell firing [20]. It has also been demonstrated that concentrations of other neurotransmitters, such as norepinephrine (NE) and serotonin (5-HT) are also elevated following MPH administration [21-23].

In addition to reducing the core ADHD symptoms, MPH treatment is often, but not inevitably, associated with a reduction in comorbid oppositional defiant disorder (ODD) and conduct disorder (CD) symptoms [24, 25]. The impact of MPH on neuropsychological functioning is complex. Whilst several studies have reported that MPH is associated with enhanced executive functioning [26] others using a double-blind randomised controlled 
methodology have found an improvement on tasks with low but not high executive demands [27]. Findings from a recent study suggest that MPH increased preference for delayed reward in ADHD patients [28]. Females are known to report markedly different subjective effects to psychostimulants than men; furthermore, they also progress to drug dependence more rapidly (Kosten et al., 1996; McCance-Katz et al., 2005). Differences in reward-related neural function between men and women have been reported (Dreher et al., 2006). One study has reported an independent main effect for age between ADHD cases and their non ADHD controls in a number of tasks which assess delay aversion [DAv; 29]. This main effect was reported to be significant on all assessment measures with children (6-12 years) having worse performance than adolescents (13 - 17 years). For example, using the The Maudsley's Index of Childhood Delay Aversion assessment [30] younger children were less likely to choose long delayed reward compared to adolescents . This is in line with DAv Theory which makes a number of specific predications about the effects of reward delay in particular contexts. These predictions are derived from the theory that delay-related effects are associated with alterations in the signalling of delayed rewards, and are compounded by an acquired motivation to escape or avoid delay. This is hypothesized to be conditioned over time in response to repeated exposure to social situations and failure in delay-rich settings experienced by children with altered delay reward signalling [31].

There are several AMF products licensed for use in ADHD; mixed amfetamine salts (Adderall), dextromethamfetamine (Desoxyn), dextroamfetamine (Dexedrine) and a dexamfetamine pro-drug lisdexamfetamine (Vyvanse). Whilst all of these medications are available in the United States, only immediate release dexamfetamine is licensed for use in Europe and even then it is widely available in the United Kingdom alone. As with MPH both IR (e.g. Adderall, Desoxyn and Dexedrine) and ER (Adderall XR) preparations are available. The dexamfetamine pro-drug lysdexamfetamine (Vyvanse) also lasts for around 12 hours and 
can be given as a once daily preparation. These agents also act by blocking DA reuptake and stimulating the release of DA into the synapse. Whilst they work in a similar way as MPH there are also differences between the two types of drugs (AMF stimulate DA release as well as block reuptake) and as a consequence a proportion of those who respond to MPH do not respond to AMF and vice versa [10]. AMF has been shown to influence neurotransmitter activity selectively in brain regions that are predominantly involved in reward processing [i.e., striatum, ventral striatum and nucleus accumbens; 28, 32].

Although they have different profiles of action across the day, when they are given in equivalent daily doses long acting formulations of MPH and AMF have been demonstrated to be equipotent compared to the IR preparations [33]. Both MPH and AMF appear to be equally effective in children with and without comorbid problems [34-36] and other disorders such as chronic fatigue syndrome [37] and depression [38]. Although both stimulants are generally well tolerated with common side effects including slight growth slowing, sleep problems and reduced appetite [39 in press], more serious side effects relating to psychiatric and cardiac problems can occur, but are rare [40, 41]. A number of non-stimulants have also been shown to be efficacious for the treatment of ADHD [42] however, they will not be discussed further as this review will focus solely on stimulants.

Predicting stimulant drug response: Given that not all patients respond to stimulant medication it would be helpful to identify which factors predict either a good response to ADHD pharmacological treatment or increase the risk of adverse events. Firstly, although normalization of symptoms does occur and is an appropriate goal for treatment [43] not everyone who responds benefits from complete symptom reduction. For example, Coghill et al. (2007) reported that whilst $70 \%$ of subjects had a robust and clinically significant response to $\mathrm{MPH}$, only $56 \%$ had post treatment scores within the normal range [27]. Secondly, a 
proportion of those who respond well to stimulant medication are unable to continue to take it due to intolerable adverse effects [41]. Furthermore, the presence of milder adverse events can result in poor compliance, reduced confidence in medication treatment by parents of young children [44] and low adherence especially during adolescence [45]. Finally, as the overall response rates to MPH and AMF are similar. For example, approximately $70 \%$ of patients respond to each independent drug and $25 \%$ respond to one but not the other, meaning that $95 \%$ respond to at least one [10]. It would be helpful for clinicians to be able to predict; (i) which patients are more likely to respond to a particular stimulant and; (ii) those who would be less likely to respond to either. Clearly, there are a number of possible biological and clinical factors that might be important, such as clinical presentation including symptom severity and genetic predisposition. Few studies have systematically studied multiple predictors of response and these have tended to report contradictory findings. For example, whilst both Taylor et al. (1987) and Buitelaar et al. (1995) found younger age to be a positive predictor of clinical response to MPH, the study of Taylor observed greater pre-treatment attentional impairment, and hyperactivity, poor performance on attentional tests lower IQ scores, and clumsiness in 'responders'. Buitelaar et al. (1995) however, found the opposite pattern of effects with respect to attentional impairment, hyperactivity and IQ, furthermore it was also revealed that responders were more likely than non-responders to have anxiety related difficulties [46]. Santosh et al. (2005) found that, compared to the DSM-IV diagnosis of combined type ADHD, the ICD-10 diagnosis of Hyperkinetic disorder, that describes a group with severe, pervasive and impairing ADHD, was predictive of a more robust response to medication than to behavioural treatments.

In the current paper we review published data examining two potential moderators of stimulant response; sex and age. 
Sex - a candidate moderator of stimulant response: Several factors make sex a candidate moderator of stimulant response in ADHD. Clinically, females differ from males in terms of the prevalence of ADHD [47]. Population based studies commonly report the prevalence of ADHD as between two and four times greater for males than it is for females [48, 49]. Interestingly, findings from clinic based studies suggest that girls with ADHD are less likely to be referred than boys, as ADHD is diagnosed between six to nine times more often in males than females $[7,50]$; whilst this is likely to reflect different patterns of comorbidity and referral biases, it does suggest that there may be considerable clinical differences between community and clinic based samples and that even more caution than usual is required when translating findings from studies of one population to the other. Consequently, it is important to be careful when interpreting the results of clinical trials that tend to draw their subjects from clinic based samples, as differences (or lack thereof) could represent a Berkson type bias [51]. Males and females have also been shown to differ with respect to clinical presentation and symptom profiles, with females being reported to experience fewer of the externalising components of the disorder [52]. Nevertheless Novik et al. (2006) found that girls were as likely as boys to receive stimulant medication as an initial treatment for ADHD in a large European observational study [53].

Sex differences have also been reported in pathophysiology. Anderson and Teicher (2000) demonstrated differences in DA receptor density between developing male and female rats whereby peri-adolescent male rats were more likely to show overproduction and subsequent pruning of striatal D2 receptors compared with periadolescent females [54]. Studies based on human samples have generally shown no differences in neuropsychological deficits between the sexes [e.g., 55]. 
Sex differences in the effects of psycho-active drugs in general [for a review see; 56] and stimulants in particular [e.g., 57, 58] are well established. There are well documented generalised differences between males and females in drug PK including drug metabolism [56], drug absorption [59] and distribution [60]. In addition, differences have also been reported in adverse drug reactions [61] and PD [PD; 62]. Certain sex-specific biological processes may also be relevant including changes in sex hormone levels [e.g., during pregnancy; 63]. Animal studies suggest that male and female brains might be organised differently to modulate an organism's response to stimulants [64]. More specifically, it has been reported that the reinforcing effects of stimulants may be stronger in females than in males [65]. There is also animal and human evidence to suggest that there may be sex differences in DAT density and clearance of extracellular DA [66-69]. As noted above the differences in D1 and D2 receptor densities (e.g., the upregulation of male but not female striatal DA receptors) parallels the early developmental appearance of motor symptoms of ADHD [70]. Whilst these results are interpreted as a possible explanation for why ADHD prevalence rates are higher in men than women, they could also be relevant to medication response. Studies of adult women have identified lower plasma concentrations of MPH after weight based dosing, relative to men [71], suggesting that some women may require higher doses of MPH to achieve the same MPH plasma concentration as men [72]. Patrick et al. reported the extent of d-MPH exposure was greater in men when the IR dl-MPH was dosed without ethanol and when ethanol was administered before MPH. A difference was also observed when dl-MPH was dosed before ethanol; however this did not reach statistical significance.

The extent of exposure (AUC) of d-MPH was significantly greater in men when the IR dlMPH was dosed without ethanol, as well as when ethanol was administered before MPH. This sex difference nearly reached significance when dl-MPH was dosed before ethanol. This 
study did not examine the efficacy of MPH treatment between males and females however it did measure sex differences in the subjective effects of MPH with or without ethanol; findings revealed that females reported greater subjective effects than males, despite having lower dl-MPH $C_{\max }$ and AUC values. Such differences in sex based sensitivity toward drug stimulating effects is discussed and it is suggested that this could constitute a differential vulnerability to MPH abuse [71].

Fluctuations have also been reported to impact stimulant efficacy in post pubertal [73] and adult females [74]. Sex differences in illicit drug use are demonstrated in the literature [for a recent review see 75]. Differences in striatal DA release following AMF administration have been reported in a reclopride positron emission tomography (PET) study in healthy adults, with males $(n=28)$ demonstrating markedly greater DA release than females $(n=15)$. In a further sample of healthy adults, hormonal fluctuations in females have also been shown to affect AMF response [76].

Age - a candidate moderator of stimulant response: There are a number of lines of evidence that suggest age should be considered as a potential moderator of stimulant effects on ADHD symptoms. It is well documented that ADHD is a lifespan condition [77] with many individuals continuing to experience symptoms and impairments from preschool through their adult years [78]. Although there are broad continuities with respect to ADHD symptoms patterns at the level of the individual case (i.e., a child with particular ADHD symptoms will often continue to experience similar symptoms throughout development), there are also often discontinuities with some children switching from one pattern of symptoms to another (e.g. from combined type to inattentive type or from hyperactive/impulsive type to combined type), in others the form of a symptom will change over time [e.g., from overt overactivity to inner restlessness; 77]. In the preschool period, symptoms of hyperactivity/impulsivity appear to predominate although this may reflect a 
relative difficulty in observing or describing symptoms of inattention in children this age rather than an absence of problems [77]. In middle childhood through adolescence the classic combined type is the most common presentation with a full range of inattentive, hyperactive and impulsive symptoms present. During adult years attentional problems are often the most obvious as hyperactivity has been reported to be transformed into internal agitation [79]. Patterns of impairment and comorbidity also vary from age-to-age [80]. ADHD treatment outcomes have been documented in the literature for childhood, adolescent and adult treatment outcome $[81,82]$. Apart from the Preschooler with ADHD Treatment (PATS) Study (Greenhill et al. 2006), little research has focused on treatment differences during the preschool period.

Age differences in response to stimulants have also been documented in the animal literature. Canese et al. (2009) found that, whilst adult rats had an expected increase of functional magnetic resonance imaging (fMRI) BOLD signal in nucleus accumbens and prefrontal cortex with no effects in the hippocampus, in adolescent rats MPH induced a marked and generalised decrease of BOLD signal. These effects occurred earlier $(<12$ minutes post dosing) in nucleus accumbens and prefrontal cortex, than they did in the hippocampus [83]. Furthermore, findings from a recent study suggest an increased threshold of behavioural activation following AMF treatment in adolescent rats, this has also been potentially related to changes in DA and the DA system during development [84]. There may also be age and sex differences in DAT binding and the relationships between this binding and verbal learning in humans; with women and younger participants having higher DA availability in caudate nucleus and better performance on verbal learning tasks [66]. Several reports have documented an age associated decline in DAT density $[69,85]$, with evidence of more functionally active DAT available in younger age groups [86]. 
Aim of the review: On the basis of these findings relating sex and age to DA function, both factors represent candidate moderators of stimulant drugs. The aim of the current review was to survey the literature to address two questions: (i) Do stimulant treatments for ADHD show efficacy and tolerability in both males and females and across age groups? And; (ii) within specific trials do age and sex moderate efficacy and tolerability of stimulants? Despite the obvious clinical importance of these questions, very few studies have addressed them directly $[73,87]$. In fact until relatively recently most studies focused on pre-pubertal boys (age 6 12 years). More recently studies have started to appear focusing on the preschool and adolescent and adult period.

\section{CURRENT REVIEW}

The current review focuses specifically on studies published over the past 20 years (between 1989 until October 2009). Databases searched included U.S. National Library of Medicine (PubMed), Medline, EMBASE, PsycINFO and ISI Web of Knowledge.

Literature of stimulant efficacy in different sex and age groups: Firstly, we identified randomised controlled trials (RCTs) of ADHD medication efficacy with; (i) male or female participants and; (ii) participants from each age sub-group (i.e., preschoolers, school age, adolescent and adult. For this literature search the following keywords were used to search databases: sex; gender; male; female; preschool; school age; adolescent; treatment; attention deficit/hyperactivity disorder (ADHD); randomised / randomized control trial (RCT); efficacy; and medication.

Literature searches for moderating effects of age and sex on stimulant response: Secondly, we searched for studies of medication efficacy that included an assessment of age or sex as a moderator of treatment outcome within their analysis. For this literature search the following keywords were used to search databases: sex; gender; age; treatment; attention 
deficit/hyperactivity disorder (ADHD); Methylphenidate (Ritalin, Concerta, Equasym, Metadate, Focalin, Medikinet, Attenta, Methylin, Rubifen, Biphentin and Daytrana); mixed amfetamine salts (Adderal); dexamfetamine (dexamfetamine, Dexedrine); and Lisdexamfetamine (Vyvanse). Relevant articles referenced in papers identified by this search were also included for review, and these in turn were used to identify additional reports. Moreover, pharmaceutical companies that currently market stimulant medication in Europe were also contacted and data was requested regarding the effects of age and sex on treatment outcome. Every effort has been made to provide as comprehensive a review as possible at the time of manuscript submission. For this second search (studies of medication efficacy that included an assessment of age or sex as a moderator of treatment outcome) a range of different designs were included (e.g., RCTs and observational studies).

\section{ARE STIMULANTS EFFICACIOUS FOR AND TOLERATED BY MALES AND FEMALES AND BY CHILDREN ACROSS THE AGE RANGE?}

Randomised controlled trials support the efficacy and tolerability of stimulants for both males and females and for patients of different ages from preschool to adulthood.

\section{Are Stimulants Efficacious and Well Tolerated by Both Sexes? Owing to the higher} prevalence of ADHD in males the majority of medication trials have primarily enrolled boys and men. As a consequence the efficacy of stimulants within predominantly male samples has been well documented in the literature [87-92] reducing both ADHD symptoms and related aggression and hostility [93]. Despite evidence that in recent years prescriptions for ADHD medications have increased at a greater rate for females than for males [45], females continue to be less likely than males to receive stimulant medication for the treatment of ADHD [73, 94]. In the UK the ratio of males to females receiving prescriptions for ADHD medications is around 66:1 which far exceeds both the gender ratio for ADHD in the population and the 
referral ratio [45]. Research into the treatment response for females with ADHD has lagged behind that in males. For the purpose of this review we were unable to find any studies that included only female participants. Although, many of the recent RCTs conducted by the pharmaceutical companies to demonstrate efficacy of the newer ER preparations have enrolled reasonable numbers of female subjects, however few have reported the outcomes for females separate from those for the males. Studies that have reported on the efficacy of stimulants in females (based on subgroup analyses of RCTs conducted in mixed sex samples) suggest that stimulants are an effective treatment for ADHD in female patients. There are also positive results for studies using MPH $[87,91]$ mixed AMF salts $[87,95]$ and lisdexamfetamine [96]. In general these studies suggest that the tolerability and safety of stimulants is similar in females and males. Further studies investigating the efficacy and safety of stimulants in females must be considered a high priority in psychopharmacology research [87].

Are stimulants efficacious and well-tolerated across the age range? Despite the vast increase in the number of clinical trials over the past decade that have examined the efficacy of stimulants in the treatment of ADHD, the majority of these studies have continued to focus on middle childhood (i.e., 6-12 years). However, studies focusing on preschoolers, adolescents and adults have started to appear.

Preschool: ADHD is increasingly being recognised as a valid disorder in the preschool period with treatment typically revolving around non-pharmacological options [7]. In most countries stimulant medications are not licensed for use in children younger than age 6 years, although dexamfetamine does have marketing authorisation for use with children as young as 3 years of age in the UK. Even though national and international guidelines for ADHD management do not recommend pharmacotherapy treatment for preschool children [6] use of stimulant 
medications for ADHD children in the preschool years (age 3 to 6 years) is becoming more commonplace, albeit from a low base.

In the United States, where the use of AMF products is more common than in Europe, MPH still accounts for over $90 \%$ of stimulant use in preschool children [97]. Only a small number of studies have investigated the efficacy of MPH in exclusively in preschool samples [98100, e.g., 101]. By far the most important and the largest of these studies is the 'Preschooler with ADHD Treatment Study [PATS; 34]. Similar to other trials this study showed that although stimulants show efficacy in the preschool years, effect sizes are somewhat smaller and the numbers needed to treat is larger than those reported in school aged children. Moreover, tolerability was reported to be reasonable, with $11 \%$ of children dropping out because of intolerable adverse effects. Overall adverse effect profiles (e.g., crying, irritability and social withdrawal) appeared to be more pronounced and occurred at smaller doses than is usually seen in older children $[102,103]$. It was also suggested that the acceptability of medication was lower in this age-group than for other age groups as retention rates were relatively low, with only 77 of the 183 preschoolers entering the medication phase of the trial [34]. Furthermore, there is particularly strong evidence of increased susceptibility to side effects for those preschool children with ADHD and comorbid developmental disabilities [100]. Although dexamfetamine is licensed for use in preschoolers in the UK we did not identify any RCTs that have specifically examined the efficacy of any AMF in preschool children.

Middle Childhood: As mentioned above the majority of the trials have enrolled male participants in middle childhood. Both MPH and AMF are generally well tolerated and efficacious in approximately $70 \%$ of cases. This evidence has been systematically reviewed on several occasions and will not be presented further [7, 104]. 
Adolescence: Adolescent ADHD represents a particular treatment challenge because of; (i) increased problems with adherence [105] and; (ii) the emergence of complicated comorbidities, such as depression [104] and substance use disorder [SUD; 106]. Surprisingly, few studies have reported the efficacy of MPH in adolescent samples [107, 108]. In part, it is possible that this reflects the difficulties (and also the potential added cost) associated with recruiting and retaining adolescents in clinical trials. The overall findings from studies in this age group suggest that MPH continues to reduce the severity of the core symptoms of ADHD in adolescence [108]. Furthermore, the adverse event profile is similar to that observed in middle childhood [109]. Studies examining AMF in adolescent samples have similarly confirmed its efficacy and tolerability in this age group [110,111]. Lack of adherence is a particular challenge in adolescence [45]. There are many potential factors which could explain this including, frustrations dealing with the occurrence of long term adverse drug reactions to stimulant use (e.g., headaches, and stomach pain [112] and/or a change in terms of demands of maintaining focus and concentration from the school setting to the workplace [105]. More evidence on medication safety and efficacy, and especially adherence and effectiveness, within adolescence is required given the high prevalence of comorbid mental disorders, multi-drug regimens and off-label use in this age group [113].

Adults: There is a growing body of evidence supporting the validity of the adult ADHD diagnosis [114] . Although the presentation of ADHD in adulthood may change, continuity has been reported between childhood, adolescent and adult ADHD. In addition to the core neurobiology also appearing to be similar, for example one study revealed smaller total cerebral brain volumes in ADHD patients from childhood through to adolescence [115] suggesting that genetic and/or environmental influences on brain development are fixed [77]. 
Both the British Association of Psychopharmacology guidelines [81] and the most recent NICE guidelines have recommended the use of stimulants for the treatment of adults with ADHD [7]. Despite this, a recent report has shown the prevalence of prescribing drugs to treat ADHD by general practitioners in the UK drops significantly for patients from age 15 to age 21 years [45] with very few individuals remaining on medication throughout this period. There are now several published RCTs which describe the efficacy of MPH in adult patients [95, 116-122]. Findings have revealed comparable effects of MPH in adults as those observed in children and adolescents $[116,120,123]$. There is also a similar safety profile [124]. One meta-analysis reported that the overall effect size of MPH treatment in adults was smaller than expected based on previous findings $(\mathrm{d}=0.42)$ in this area [122]; furthermore, this is also significantly lower than what has been generally reported in childhood. Fewer trials have been conducted which examine the role of AMF for the treatment of adult ADHD [125-127]. In general, these studies suggest that AMF is efficacious and is well tolerated in adult ADHD patients, with most treatment emergent adverse effects being of mild to moderate severity [127].

\section{DO SEX AND AGE MODERATE THE DEGREE OF STIMULANT RESPONSE?}

There is good evidence that both MPH and AMF are efficacious and tolerated by both males and females, and by patients of different ages. Here we turn to the question of whether there is evidence that the degree of efficacy or extent of adverse events varies by age and sex within studies. To answer this question it is important to identify studies that have sufficient power and range of participants of different ages and sex to allow a within subject statistical analysis. Unfortunately such studies are uncommon.

Is there any evidence that sex is a moderator of stimulant response? The majority of research that has examined sex as a moderator of treatment response has focused on MPH. 
Nine RCTs have been identified from the literature that examined the effect of a participant's sex on stimulant response $[10,87,91,96,128-131]$. Three studies examined the effects of IR MPH. The Multimodal Treatment of Children With ADHD study [90], compared 114 female ADHD patients and 165 male patients, aged 7-9 years [132]. This study reported no significant effects of sex as a moderator of drug effects on either symptom reduction or side effects. Barkley (1989) examined the effects of MPH on mother-child interactions in a sample of 20 girls and 20 boys (age 3 years 10 months - 10 years 1 month). The results of this study revealed no significant interactions between the sex and the dose effects of medication [130]. One study examined the effects of MPH on a sample of 12 boys (aged 5 years 6 months -11 years) and 12 girls [aged 5 years 8 months -11 years 3 months; 129]. The results of this study revealed that girls responded to MPH in the same manner as an age and IQ matched group of boys.

Three studies have looked at the moderating effects of sex on the efficacy of long acting MPH preparations; one in children [131] one in adolescents and young adults [87] and one in adults [128]. The Sonuga-Barke et al. (2007) study is based on the COMACS data, which is a study of the comparison of once-daily ER MPH formulations in children with ADHD in the laboratory school [17]. In this original study assessments were made by two trained observers during classroom sessions on the basis of a 1.5-hour cycle of activities, with separate assessments of attention and deportment being made at $0,1.5,3.0,4.5,6.0,7.5$, and then 12 hours following drug administration. In a reanalysis of this study data, Sonuga-Barke et al. (2007) compared 136 boys and 48 girls (aged 6 - 12 years) in a double-blind cross over trial of Concerta, MetadateCD/Equasym XL, or placebo, using a laboratory classroom setting with measurements of symptom change by trained observers across the day and overall response over the 7 day period as rated by the children's parents using the Swanson, Nelson, and Pelham scale version IV [SNAP-IV; 133]. The results of this study revealed there was no 
main effect of sex on either measure of overall response. Both sexes responded to MPH equally well however it remained possible that differential DA function and sex related PK differences could lead to different patterns of effect across the day, even where the average control across the whole day is the same. Interestingly compared with males, females had a statistically superior response at 1.5 hours after drug administration and an inferior response at the 12 hour time point [131]. A more recent study in a sample of adolescents (aged $16-18$ years) reported no sex differences in treatment response to either osmotic release MPH $\left(\mathrm{OROS}^{\circledR} \mathrm{MPH}\right)$ and mixed AMF salts [se-AMF ER; 87]. In this study, response to medication was assessed for 16 females and 19 males using adolescent and parent ADHD rating scales (Conners Brief Rating Scale Revised, objective measures of inattention and hyperactive impulsive errors during a driving simulator task), neuropsychological tasks (Go/No-go test and the Delayed Matching-to-Sample Task) and an adolescent side effects rating scale (Stimulant Side Effects Rating Scale). No sex differences were evident on any measure although it is questionable whether the sample sizes were large enough to support a negative finding [87]. Ramos-Quiroga et al. (2009) investigated the predictors of response in a secondary analysis of data from a large RCT of long acting MPH in a group of adults $(\mathrm{n}=$ 401) aged between 18 - 65 years of age, with a high proportion of female participants (46\%). Unlike the other studies they found that whilst as a group both sexes responded better to MPH than placebo, being male was associated with a superior response at the end of the double blind phase of the study [128]. Two studies examined the effects of sex on response to both MPH and dexamfetamine (DEX) treatment of children with ADHD [91]. Efron and colleagues (1997) examined a group of 114 boys (mean age 105 months) and 11 girls (mean age 102.4 months). The findings from this study revealed that sex was not predictive of response to either stimulant [10]. One further study examined the effects of both MPH and DEX in a sample of 42 girls (age 6.2 - 12.7 years) and 56 boys [age 6.0 to 12.5 years; 91 ]. 
The results of this study confirmed previous findings that females are comparable to males in terms of their response to treatment with both stimulants; with $95 \%$ of females responding well to one or both drugs. Finally a recently completed lab-school study of lisdexamfetamine also concluded that there were no differences in overall response between male and female subjects [96].

As the studies by Sonuga-Barke et al. (2007) and by Wigal et al. (2009) employed a lab classroom design with multiple measures of efficacy across the day, it was possible to examine whether sex impacted on MPH and AMF PD. Wigal et al. (2009) employed an almost identical lab school design except that measures were taken at -0.5 hours pre-dose and then at $1.5,2.5,5.0,7.5,10.0,12.0$, and 13.0 hours post-dose. However, this study found no sex differences with respect to PD of lisdexamfetamine. These results should be interpreted with caution due to the relatively small number of female participants ( 28 out of a total sample of 113 participants) compared to the somewhat larger Sonuga-Barke et al. (2007) sample (48 of a total of 184 participants).

\section{Is there any evidence that age is a moderator of stimulant effects?}

Few clinical trials have included a sufficiently broad age range to address this question adequately with most studies recruiting only in middle childhood (around the $6-12$ year age range). However, six studies were identified from the literature that directly examined age as a moderator of stimulant treatment outcome in children and adolescents albeit often across a narrow age range $[27,46,111,134-136]$ and one in adults [128]. Whilst the findings from these studies are somewhat inconsistent, this is probably a consequence of the small numbers of participants in many studies and the limited age ranges of some. Taylor et al. (1987) conducted a double-blind, placebo-controlled, crossover trial of MPH and placebo with thirty-eight boys (6 - 10 years of age), referred for psychiatric treatment due to serious 
behaviour problems. As not all subjects had either an ICD 9 or DSM III diagnosis of ADHD some caution is advised when interpreting the results. A good response was predicted by younger age as well as higher levels of inattentive and restless behaviour, impaired performance on tests of attention, clumsiness, and the absence of symptoms of overt emotional disorder. Diagnostic status (ICD 9 or DSM III) was not found to be a good predictor of MPH response [135]. Using a robust measure of response, Buitelaar et al. (1995) also found that young age predicted a stronger response to MPH in 46 children (aged between 6 and 13 years). Strong response was also predicted by high IQ, considerable inattentiveness, low severity of disorder, and low rates of anxiety [46]. Using the same measures of response Coghill et al. (2007) did not find an effect of age on any measure of response in $3 \mathrm{x} 4$ week placebo-controlled, double-blinded, randomised, crossover trial of two dose levels of MPH ( 0.3 and $0.6 \mathrm{mg} / \mathrm{kg} /$ dose given twice daily and placebo) in 75 boys, aged $7-15$ years with hyperkinetic disorder [27]. Pelham and colleagues (1991) compared the effectiveness of MPH treatment in a sample of all male children, 17 were between the ages 7 years 3 months to 10 years 11 months and a further 17 were adolescents between the ages of 12 years to 14 years and 6 months. The findings of this study revealed treatment efficacy and tolerability to be similar in both age groups [136]. Findling and colleagues (2001) replicated these findings for both MPH and AMF in a randomised controlled crossover trial of these agents in 69 young children (aged from 4 years to 7.99 years), 56 youths (aged from 8 years to 10.99 years) and 52 adolescents (aged from 11 years to 17.59 years); however, whilst both agents had similar efficacy in young children and adolescents, youths were found to benefit from smaller weight-adjusted doses than did the younger children [111]. In the study of RamosQuiroga et al. (2009) described earlier, at the end of the double blind phase of the trial, older age (> 45 years) was associated with better treatment outcomes with long acting MPH than younger age $[18-45$ years; 128$]$. 


\section{DISCUSSION}

In this review we have investigated the evidence relating to the moderating effects of sex and age on the response of individuals with ADHD to stimulant medications. Such effects may be expected as a consequence of the sex and age related differences that have been shown in relation to DA function [69, 86, 137]. Two important general findings are: (i) notwithstanding the large number of clinical trials and other studies that support the efficacy, effectiveness, safety and tolerability of the stimulants MPH and AMF in the treatment of ADHD [7], relatively few studies have described the effects of sex and age on any of these outcomes; and (ii) taken together studies that have examined such effects suggest that stimulant medications are equally efficacious, effective, well tolerated by both males and females and by individuals from childhood through to adulthood: Evidence to date suggests that sex and age play a minimal role in moderating overall efficacy and safety of stimulants however further research is required in this area.

These are important findings as ADHD is being increasingly recognised in females, adolescents and adults. Furthermore, whilst many of those with ADHD in these groups would benefit from treatment with stimulants, such treatment is (within Europe at least) relatively uncommon at the present time. The main proviso to this general message is that stimulants seem to be less efficacious for preschoolers and side-effects may be greater in this age group. The strongest evidence for this can be derived from the PATS study [e.g., PATS; 34] in which the effect sizes were smaller and there were more pronounced adverse effects occurred at smaller doses than is generally seen in studies with older subjects. The clinical message here seems clear; in accordance with clinical guidelines clinicians should consider using stimulants to treat ADHD in children from around the age of 6 years through to adulthood irrespective of age or sex. Stimulants should be used with caution in those under six years of age and probably reserved for those with severely impairing symptoms that have failed to 
respond to non-pharmacological treatments. This last evidence-based recommendation also makes clinical sense as even though ADHD symptoms are usually apparent very early in life it is much more difficult to make a firm diagnosis of ADHD in the preschool child. It is difficult to recognise inattentive behaviours in preschoolers and there is a far wider variation in normal behaviour at this age. It is also the case that non-pharmacological behavioural interventions have been demonstrated to be effective in this age group and are likely to be safer especially where diagnostic uncertainty is present $[138,139]$.

Evidence suggests that, apart from in the preschool period, there are few age differences in response and tolerability for either MPH or AMP during childhood or adolescence and into early adulthood. Whilst both Taylor et al. (1987) and Buitelaar et al. (1995) found that younger age was associated with a better response these studies included relatively small samples with restricted age ranges. Neither of the two larger studies in children and young people [27, 111] found an effect of age on response. The finding reported by Ramos-Quiroga et al. (2009) that in their adult sample, the factors of older age and being male was associated with a better response are intriguing. This was a large study with a very broad range of ages and a high proportion of female subjects and clearly the results need to be taken seriously. Whilst these general recommendations may come as no surprise to many, they are important as they do not seem to reflect current routine clinical practice with respect to either sex or age. Data suggests that on top of the referral bias that sees boys with ADHD being more likely to be diagnosed than girls it seems that females that are diagnosed are less likely to be treated with stimulant medication than are their male counterparts [53]. Observational studies in clinical samples suggest that girls in Europe who are diagnosed with ADHD are as likely as boys to be prescribed stimulant medication as a first treatment [53]. Prescribing databases in the UK suggest that females are much less likely to be receiving prescriptions for stimulant medications than males with a ratio of male to female of $66: 1$ [45]. It is only possible to 
speculate why this situation may have arisen. As girls often present with more inattentive symptoms and less oppositional and disruptive behaviours some clinicians may be reluctant to treat with stimulants mistakenly believing them only to be effective against the hyperactive and impulsive behaviours. It is also possible that whilst clinicians are recommending medication, it is the patients and/or their parents who themselves feel medication is less relevant for the types of problems girls are presenting with.

Whilst the evidence presented in this review seems to make it unlikely that differential response rates or adverse effects could account for these differences, it is clear that stimulants work well in both sexes. However, the findings of Sonuga Barke et al. (2007) highlight that overall response is only one aspect of successful treatment. Ideally treatment should be effective across as much of the day as possible. The introduction of the lab school protocol has allowed the PD of stimulant effects to be measured within a clinical trial. The data reported by Sonuga-Barke et al. (2007) suggest another possible route by which females may be 'undertreated' and less happy with their medication. This study reported that compared to males, females have a stronger early response to MPH, but that this effect also wears off more quickly in females than males. The reasons for these sex differences are unclear but may be related to variations in sex differences in metabolism of MPH, the PK profile of MPH or differences in DA functioning between males and females [68]. These early and late day differences in drug effect were found to be independent of the two ER MPH preparations and suggest that females may require a different dosing strategy than males with a larger dose in the late afternoon. It is likely that working at such a level of detail regarding the profile of effects across the day is not a part of routine clinical practice. As is the case in clinical trials, clinicians are often asking the simpler question "is medication working?" rather than "is this medication working optimally across the day?" It is possible that such an approach would lead to a proportion of female patients who are well treated during the day having increased 
difficulties at home in the late afternoon and evenings. In turn this could result in dissatisfaction with treatment, and for the patient and their family, an early discontinuation of a potentially helpful treatment. Author DC has developed a clinical tool, the Dundee Difficult Times of Day Scale (DDTODS; available from DC) that can help the clinician assess adequacy of treatment across the day and adjust treatment accordingly. It is interesting that the study of lisdexamfetamine, conducted by Wigal et al. (2009), which also used a labschool design to investigate sex effects did not find any differences between male and female participants with this AMF-pro drug. It is possible that this is a reflection of the interaction between sex and the different metabolic pathways for AMF and MPH drugs. AMF are primarily metabolized by the cytochrom-P450 isoenzyme 2D6 and MPH by carboxylesterase 1A. The findings of both Sonuga-Barke et al. (2007) and Wigal et al. (2009) clearly need to be replicated and if the results are confirmed the reasons for the differences need to be carefully explored.

When interpreting these findings we need to be aware of inherent limitations in the available literature. First, it has been noted already that very few studies were designed to address the issue of sex and age-related effects on stimulant response. This means that few studies were sufficiently powered to test for age and sex effects. In particular, they did not contain a sufficiently wide range of ages or a large enough number of female patients. Even where secondary analyses of the effects of sex and age were possible (as is the case for some of the recent large scale registration trials conducted by the pharmaceutical companies), there has often been a reluctance on the part of the companies to engage in the study of predictors of treatment response because of concern about potential product 'niching' (i.e., identifying subgroups for whom the project works best). Second, there are far fewer studies looking specifically at AMF products with regard to age and sex effects. Third, the outcome measures used in many studies have been rather limited in their range. The outcomes commonly used 
were usually limited to parental, nursery/teacher reports on ADHD rating scales, with few examples of objective behavioural observations of core symptoms, standardised laboratory psychological tests or patient reported outcomes relating to broader outcomes such as quality of life [140]. It is possible that sex and age effects may emerge with different levels of measurement other core symptom changes. Fourth, few studies have employed methodologies that allow sex and age-related differences in PD to be examined. As can be seen from the lab school study reported by Sonuga-Barke et al. (2007) such studies have the power to identify more subtle differences between groups than is possible with the more general measures taken in most clinical trials. Finally, the effects of comorbidity as a confounding factor and/or a secondary outcome in sex and age effects have not been addressed to date.

The key challenge for the future is therefore to design studies specifically to test the sex and age moderation hypotheses across a range of different stimulant and non-stimulant medications with equal numbers of male and female patients stratified for age and stage of development, and including measures relating to PD, neuropsychological functioning and quality of life as well as direct observation and symptom scales. Smaller scale studies should include PK and DA function measures and sex related biomarkers (such as hormonal levels and cycles) to examine the extent to which sex and age-related neurochemical effects mediate any clinical differences that might be observed. 


\section{REFERENCES}

1. Taylor, E. and Sonuga-Barke, E., Disorders of attention and activity, in Rutter's Child and Adolescent Psychiatry, M. Rutter, et al., Editors. 2008, Blackwell-Wiley: Oxford.

2. Satterfield, J. and Schell, A., A prospective study of hyperactive boys with conduct problems and normal boys: adolescent and adult criminality. Journal of the American Academy of Child \& Adolescent Psychiatry, 1997. 36(12): p. 1726-35.

3. Swanson, J., Swartz, M., et al., Psychiatric impairment, social contact, and violent behavior: evidence from a study of outpatient-committed persons with severe mental disorder. Social Psychiatry \& Psychiatric Epidemiology, 1998. 33 Suppl 1: p. S8694.

4. Biederman, J., Mick, E., et al., Normalized functioning in youths with persistent attention-deficit/hyperactivity disorder. Journal of Pediatrics, 1998. 133(4): p. 544-51.

5. Schubiner, H., Tzelepis, A., et al., Prevalence of attention-deficit/hyperactivity disorder and conduct disorder among substance abusers. Journal of Clinical Psychiatry, 2000. 61(4): p. 244-51.

6. Banaschewski, T., Coghill, D., et al., Long-acting medications for the hyperkinetic disorders. A systematic review and European treatment guideline. European Child \& Adolescent Psychiatry, 2006. 15(8): p. 476-95.

7. NICE, Attention Deficit Hyperactivity Disorder: Diagnosis and Management of ADHD in children, Young People and Adults. 2008, National Institute for Health and Clinical Excellence.

8. Barkley, R., Adolescents with attention-deficit/hyperactivity disorder: an overview of empirically based treatments. Journal of Psychiatric Practice, 2004. 10(1): p. 39-56.

9. Spencer, T., Biederman, J., et al., Pharmacotherapy of attention-deficit hyperactivity disorder across the life cycle. Journal of the American Academy of Child \& Adolescent Psychiatry, 1996. 35(4): p. 409-32. 
10. Efron, D., Jarman, F., et al., Methylphenidate versus dexamphetamine in children with attention deficit hyperactivity disorder: A double-blind, crossover trial. Pediatrics, 1997. 100(6): p. E6.

11. Carlson, P.J., Merlock, M.C., et al., Adjunctive stimulant use in patients with bipolar disorder: treatment of residual depression and sedation. Bipolar Disorders, 2004. 6(5): p. 416-420.

12. Mitler, M.M., Erman, M., et al., Amphetamines and narcolepsy. The treatment of excessive somnolence with stimulant drugs. . Sleep, 1993. 16(3): p. 203-206.

13. Swanson, J. and Volkow, N., Pharmacokinetic and pharmacodynamic properties of methylphenidate in humans. Stimulant drugs and ADHD: Basic and clinical neuroscience. 2001, New York: Oxford University Press. 259-282.

14. Kimko, H.C., Cross, J.T., et al., Pharmacokinetics and clinical effectiveness of methylphenidate. Clinical Pharmacokinetics, 1999. 37(6): p. 457-70.

15. Anderson, V.R. and Scott, L.J., Methylphenidate transdermal system - In attentiondeficit hyperactivity disorder in children. Drugs, 2006. 66(8): p. 1117-1126.

16. Sonuga-Barke, E.J.S., Swanson, J.M., et al., Efficacy of two once-daily methylphenidate formulations compared across dose levels at different times of the day: Preliminary indications from a secondary analysis of the COMACS study data. BMC Psychiatry, 2004. 4(30).

17. Swanson, J., Wigal, S.B., et al., A comparison of once-daily extended-release methylphenidate formulations in children with attention-deficit/hyperactivity disorder in the laboratory school (the Comacs Study). Pediatrics, 2004. 113: p. 206-16.

18. Volkow, N.D., Wang, G.J., et al., Imaging the effect of methylphenidate on brain dopamine: New model on its therapeutic actions for attention-deficit/hyperactivity disorder. Biological Psychiatry, 2005. 57(11): p. 1410-1415.

19. Grace, A.A., Psychostimulant actions on dopamine and limbic system function: Relevance to the pathophysiology and treatment of ADHD. 2001, New York, NY: 
Oxford University Press. (2001). Stimulant drugs and ADHD: Basic and clinical neuroscience. (pp. 134-157). xii, 410.

20. Volkow, N.D., Wang, G., et al., Therapeutic doses of oral methylphenidate significantly increase extracellular dopamine in the human brain. Journal of Neuroscience, 2001. 21(2).

21. Arnsten, A.F. and Li, B.M., Neurobiology of executive functions: catecholamine influences on prefrontal cortical functions. Biological Psychiatry, 2005. 57(11): p. 1377-84.

22. Kuczenski, R. and Segal, D., Effects of methylphenidate on extracellular dopamine, serotonin, and norepinephrine: comparison with amphetamine. Journal of Neurochemistry, 1997. 68(5): p. 2032-7.

23. Solanto, M.V., Neuropsychopharmacological mechanisms of stimulant drug action in attention-deficit hyperactivity disorder: a review and integration. Behavioural Brain Research, 1998. 94(1): p. 127-52.

24. Klein, R.G., Abikoff, H., et al., Clinical efficacy of methylphenidate in conduct disorder with and without attention deficit hyperactivity disorder. Archives of General Psychiatry, 1997. 54(12): p. 1073-1080.

25. Serra-Pinheiro, M.A., Mattos, P., et al., The effect of methylphenidate on oppositional defiant disorder comorbid with attention deficit/hyperactivity disorder. Arquivos De Neuro-Psiquiatria, 2004. 62(2B): p. 399-402.

26. Mehta, M.A., Owen, A.M., et al., Methylphenidate enhances working memory by modulating discrete frontal and parietal lobe regions in the human brain. Journal of Neuroscience, 2000. 20(6).

27. Coghill, D.R., Rhodes, S.M., et al., The Neuropsychological Effects of Chronic Methylphenidate on Drug-Naive Boys with Attention-Deficit/Hyperactivity Disorder. Biological Psychiatry, 2007. 62(9): p. 954-962. 
28. Shiels, K., Hawk, L.W., Jr., et al., Effects of methylphenidate on discounting of delayed rewards in attention deficit/hyperactivity disorder. Experimental and Clinical Psychopharmacology, 2009. 17(5): p. 291-301.

29. Bitsakou, P., Psychogiou, L., et al., Delay Aversion in Attention Deficit/Hyperactivity Disorder: an empirical investigation of the broader phenotype. Neuropsychologia, 2009. 47(2): p. 446-56.

30. Kuntsi, J. and Stevenson, J., Psychological mechanisms in hyperactivity: II. The role of genetic factors. Journal of Child Psychology \& Psychiatry \& Allied Disciplines, 2001. 42(2): p. 211-9.

31. Sonuga-Barke, E.J.S., The dual pathway model of AD/HD: an elaboration of neurodevelopmental characteristics. Neuroscience \& Biobehavioral Reviews, 2003. 27(7): p. 593-604.

32. Drevets, W.C., Gautier, C., et al., Amphetamine-induced dopamine release in human ventral striatum correlates with euphoria. Biological Psychiatry, 2001. 49(2): p. 8196.

33. Findling, R.L., McNamara, N.K., et al., Combination lithium and divalproex sodium in pediatric bipolar symptom restabilization. Journal of the American Academy of Child and Adolescent Psychiatry, 2006. 45(2): p. 142-148.

34. Greenhill, L., Kollins, S., et al., Efficacy and safety of immediate-release methylphenidate treatment for preschoolers with ADHD. Journal of the American Academy of Child and Adolescent Psychiatry, 2006. 45(11): p. 1284-1293.

35. Gadow, K.D., Nolan, E.E., et al., Methylphenidate in children with oppositional defiant disorder and both comorbid chronic multiple tic disorder and ADHD. Journal of Child Neurology, 2008. 23(9): p. 981-990.

36. Najib, J., The Efficacy and Safety Profile of Lisdexamfetamine Dimesylate, a Prodrug of d-Amphetamine, for the Treatment of Attention-Deficit/Hyperactivity Disorder in Children and Adults. Clinical Therapeutics, 2009. 31(1): p. 142-176. 
37. Blockmans, D., Persoons, P., et al., Does methylphenidate reduce the symptoms of chronic fatigue syndrome? Am J Med, 2006. 119(2): p. 167.e23-30.

38. Huang, C.C., Shiah, I.S., et al. Adjunctive use of methylphenidate in the treatment of psychotic unipolar depression. in 3rd Collegium International NeuroPsychopharmacologium, Asia Pacific Regional Meeting. 2007. Bangkok, THAILAND: Lippincott Williams \& Wilkins.

39. Sonuga-Barke, E.J.S., Coghill, D., et al., Adverse reactions to methylphenidate treatment for ADHD: Structure and associations with clinical characteristics and symptom control. Journal of Child and Adolescent Psychopharmacology. in press

40. Winterstein, A.G., Gerhard, T., et al. Cardiac Safety of Methylphenidate Versus Amphetamine Salts in the Treatment of ADHD. in 23rd International Conference on Pharmacoepidemiology and Therapeutic Risk Management. 2007. Quebec City, CANADA: Amer Acad Pediatrics.

41. Graham, J. and Coghill, D., Adverse effects of pharmacotherapies for attention-deficit hyperactivity disorder: Epidemiology, prevention and management. CNS Drugs, 2008. 22(3): p. 213-237.

42. Cheng, J.Y., Chen, R.Y., et al., Efficacy and safety of atomoxetine for attentiondeficit/hyperactivity disorder in children and adolescents-meta-analysis and metaregression analysis. Psychopharmacology, 2007. 194(2): p. 197-209.

43. Steele, M., Jensen, P.S., et al., Remission versus response as the goal of therapy in ADHD: A new standard for the field? Clinical Therapeutics, 2006. 28(11): p. 18921908.

44. dosReis, S., Butz, A., et al., Attitudes about stimulant medication for attentiondeficit/hyperactivity disorder among African American families in an inner city community. Journal of Behavioral Health Services \& Research, 2006. 33(4): p. 42330. 
45. McCarthy, S., Asherson, P., et al., Attention-deficit hyperactivity disorder: treatment discontinuation in adolescents and young adults. British Journal of Psychiatry, 2009. 194(3): p. 273-7.

46. Buitelaar, J.K., Vandergaag, R.J., et al., Prediction of clinical response to methylphenidate in children with attention deficit hyperactivity disorder. . Journal of the American Academy of Child and Adolescent Psychiatry, 1995. 34(8): p. 10251032.

47. Gomez, R., Harvey, J., et al., DSM-IV AD/HD: Confirmatory factor models, prevalence, and gender and age differences based on parent and teacher ratings of Australian primary school children. Journal of Child Psychology and Psychiatry and Allied Disciplines, 1999. 40(2): p. 265-274.

48. Mukhopadhyay, M., Misra, S., et al., Attention Deficit Hyperactivity Disorder. Indian Journal of Pediatrics, 2003. 70(10): p. 789-792.

49. St Sauver, J.L., Barbaresi, W.J., et al., Early life risk factors for attentiondeficit/hyperactivity disorder: a population-based cohort study. Mayo Clinic Proceedings, 2004. 79(9): p. 1124-31.

50. NHS Quality Improvement Scotland, in ADHD Service Over Scotland. 2008: Edinburgh.

51. Berkson, J., Limitations of the application of the fourfold table analysis to hospital data. Biometrics, 1946. 2: p. 47-53.

52. Gaub, M. and Carlson, C.L., Gender differences in ADHD: A meta-analysis and critical review. Journal of the American Academy of Child and Adolescent Psychiatry, 1997. 36(8): p. 1036-1045.

53. Novik, T.S., Hervas, A., et al., Influence of gender on attention-deficit/hyperactivity disorder in Europe - ADORE. European Child and Adolescent Psychiatry, 2006. 15(1): p. I/15-I/24.

54. Andersen, S.L. and Teicher, M.H., Sex differences in dopamine receptors and their relevance to $A D H D$. Neuroscience \& Biobehavioral Reviews, 2000. 24(1): p. 137-41. 
55. Rucklidge, J.J. and Tannock, R., Neuropsychological profiles of adolescents with ADHD: effects of reading difficulties and gender. Journal of Child Psychology \& Psychiatry \& Allied Disciplines, 2002. 43(8): p. 988-1003.

56. Craft, R., Sex differences in drug- and non-drug-induced analgesia. Life Sciences, 2003. 72(24): p. 2675-2688.

57. Kosten, T., Gawin, F., et al., Gender differences in cocaine use and treatment response. Journal of Substance Abuse Treatment, 1993. 10(1): p. 63-66.

58. Booze, R.M., Wood, M.L., et al., Estrous cyclicity and behavioral sensitization in female rats following repeated intravenous cocaine administration. Pharmacology Biochemistry and Behavior, 1999. 64(3): p. 605-610.

59. Gorski, J.C., Vannaprasaht, S., et al., The effect of age, sex, and rifampin administration on intestinal and hepatic cytochrome P450 3A activity. Clinical Pharmacology and Therapeutics, 2003. 74(3): p. 275-287.

60. Sowinski, K.M., Abel, S.R., et al., Effect of gender on the pharmacokinetics of ofloxacin. Pharmacotherapy, 1999. 19(4 I): p. 442-446.

61. US General Accounting Office, ed. U.G.A.O.A.D. Events. 2000, Washington DC.

62. Rathore, S.S., Wang, Y., et al., Sex-based differences in the effect of digoxin for the treatment of heart failure. New England Journal of Medicine, 2002. 347(18): p. 14031411.

63. Loebstein, R., Lalkin, A., et al., Pharmacokinetic changes during pregnancy and their clinical relevance. Clinical Pharmacokinetics, 1997. 33(5): p. 328-343.

64. Hu, M., Crombag, H.S., et al., Biological basis of sex differences in the propensity to self-administer cocaine. Neuropsychopharmacology, 2004. 29(1): p. 81-5.

65. Roth, M.E. and Carroll, M.E., Sex differences in the escalation of intravenous cocaine intake following long- or short-access to cocaine self-administration. Pharmacology Biochemistry and Behavior, 2004. 78(2): p. 199-207. 
66. Mozley, L.H., Gur, R.C., et al., Striatal dopamine transporters and cognitive functioning in healthy men and women. American Journal of Psychiatry, 2001. 158(9): p. 1492-1499.

67. Rivest, R., Falardeau, P., et al., Brain dopamine transporter - gender differences and effect of chronic haloperidol. . Brain Research, 1995. 692(1-2): p. 269-272.

68. Bhatt, S.D., Dluzen, D.E., et al., Dopamine transporter function differences between male and female CD-1 mice. Brain Research, 2005. 1035(2): p. 188-95.

69. Lavalaye, J., Booij, J., et al., Effect of age and gender on dopamine transporter imaging with [I-123]FP-CIT SPET in healthy volunteers. European Journal of Nuclear Medicine, 2000. 27(7): p. 867-869.

70. Andersen, S.L., Dumont, N.L., et al., Differences in behavior and monoamine laterality following neonatal clomipramine treatment. Developmental Psychobiology, 2002. 41(1): p. 50-7.

71. Patrick, K.S., Straughn, A.B., et al., Influence of ethanol and gender on methylphenidate pharmacokinetics and pharmacodynamics. Clinical Pharmacology \& Therapeutics, 2007. 81(3): p. 346-353.

72. Markowitz, J.S., Straughn, A.B., et al., Advances in the pharmacotherapy of attention-deficit-hyperactivity disorder: focus on methylphenidate formulations. Pharmacotherapy, 2003. 23(10): p. 1281-99.

73. Quinn, P.O., Treating adolescent girls and women with ADHD: gender-specific issues. Journal of Clinical Psychology, 2005. 61(5): p. 579-87.

74. Justice, A.J.H. and de Wit, H., Acute effects of estradiol pretreatment on the response to d-amphetamine in women. Neuroendocrinology, 2000. 71(1): p. 51-59.

75. Dluzen, D.E., Liu, B., et al., Gender differences in methamphetamine use and responses: a review. Gender Medicine, 2008. 5(1): p. 24-35.

76. White, T.L., Justice, A.J., et al., Differential subjective effects of D-amphetamine by gender, hormone levels and menstrual cycle phase. Pharmacology, Biochemistry \& Behavior, 2002. 73(4): p. 729-41. 
77. Spencer, T., Biederman, J., et al., Attention-deficit/hyperactivity disorder: Diagnosis, lifespan, comorbidities, and neurobiology. Journal of Pediatric Psychology, 2007. 32(6): p. 631-642.

78. Biederman, J., Faraone, S., et al., Diagnostic continuity between child and adolescent ADHD: Findings from a longitudinal clinical sample. Journal of the American Academy of Child \& Adolescent Psychiatry, 1998. 37(3): p. 305-313.

79. Mannuzza, S., Klein, R., et al., Adult psychiatric status of hyperactive boys grown up. American Journal of Psychiatry, 1998. 155(4): p. 493-498.

80. Wilens, T., Biederman, J., et al., Attention deficit/hyperactivity disorder across the lifespan. Annual Review of Medicine, 2002. 53: p. 113-131.

81. Nutt, D.J., Fone, K., et al., Evidence-based guidelines for management of attentiondeficit/hyperactivity disorder in adolescents in transition to adult services and in adults: Recommendations from the British Association for Psychopharmacology. Journal of Psychopharmacology, 2007. 21(1): p. 10-41.

82. Molina, B.S., Hinshaw, S.P., et al., The MTA at 8 years: prospective follow-up of children treated for combined-type ADHD in a multisite study. Journal of the American Academy of Child \& Adolescent Psychiatry, 2009. 48(5): p. 484-500.

83. Canese, R., Adriani, W., et al., Peculiar response to methylphenidate in adolescent compared to adult rats: A phMRI study. Psychopharmacology, 2009. 203(1): p. 143153.

84. Mathews, I.Z., Waters, P., et al., Changes in Hyporesponsiveness to Acute Amphetamine and Age Differences in Tyrosine Hydroxylase Immunoreactivity in the Brain Over Adolescence in Male and Female Rats. Developmental Psychobiology, 2009. 51(5): p. 417-428.

85. Van Dyck, C.H., Seibyl, J.P., et al., Age-related decline in striatal dopamine transporter binding with iodine-123-beta-CIT SPECT. Journal of Nuclear Medicine, 1995. 36(7): p. 1175-1181. 
86. Volz, T.J., Farnsworth, S.J., et al., Age-Dependent Differences in Dopamine Transporter and Vesicular Monoamine Transporter-2 Function and Their Implications for Methamphetamine Neurotoxicity. Synapse, 2009. 63(2): p. 147-151.

87. Mikami, A.Y., Cox, D.J., et al., Sex Differences in Effectiveness of Extended-Release Stimulant Medication among Adolescents with Attention-Deficit/Hyperactivity Disorder. Journal of Clinical Psychology in Medical Settings, 2009. 16(3): p. 233242.

88. Elia, J., Borcherding, B.G., et al., Methylphenidate and dextroamphetamine treatments of hyperactivity - are there true nonresponders? Psychiatry Research, 1991. 36(2): p. 141-155.

89. Elia, J., Welsh, P.A., et al., Classroom academic performance: Improvement with both methylphenidate and dextroamphetamine in ADHD boys. Journal of Child Psychology and Psychiatry and Allied Disciplines, 1993. 34(5): p. 785-804.

90. MTA, Moderators and mediators of treatment response for children with attentiondeficit/hyperactivity disorder: the Multimodal Treatment Study of children with Attention-deficit/hyperactivity disorder. Archives of General Psychiatry, 1999. 56(12): p. 1088-96.

91. Sharp, W.S., Walter, J.M., et al., ADHD in girls: clinical comparability of a research sample. Journal of the American Academy of Child \& Adolescent Psychiatry, 1999. 38(1): p. 40-7.

92. Starr, H.L. and Kemner, J., Multicenter, randomized, open-label study of OROS methylphenidate versus atomoxetine: treatment outcomes in African-American children with ADHD. Journal of the National Medical Association, 2005. 97(10 Suppl): p. 11S-16S.

93. Amery, B., Minichiello, M.D., et al., Aggression in hyperactive boys: Response to damphetamine. Journal of the American Academy of Child Psychiatry, 1984. 23(3): p. 291-294. 
94. Barbaresi, W.J., Katusic, S.K., et al., Long-term stimulant medication treatment of attention-deficit/hyperactivity disorder: results from a population-based study. Journal of Developmental \& Behavioral Pediatrics, 2006. 27(1): p. 1-10.

95. Biederman, J., Wigal, S.B., et al., A post hoc subgroup analysis of an 18-day randomized controlled trial comparing the tolerability and efficacy of mixed amphetamine salts extended release and atomoxetine in school-age girls with attention-deficit/hyperactivity disorder. Clinical Therapeutics, 2006. 28(2): p. 280293.

96. Wigal, T., Kollins, S., et al., The effect of gender on treatment outcomes over 13 hours in an analog classroom study of children with attention deficit hyperactivity disorder treated with lisdexamfetamine dimesylate. US Psychiatric and Mental Health Congress Conference., 2009.

97. Zito, J.M., Safer, D.J., et al., Trends in the prescribing of psychotropic medications to preschoolers. Jama-Journal of the American Medical Association, 2000. 283(8): p. 1025-1030.

98. Kollins, S., Greenhill, L., et al., Rationale, design, and methods of the Preschool ADHD Treatment Study (PATS). Journal of the American Academy of Child and Adolescent Psychiatry, 2006. 45(11): p. 1275-1283.

99. Firestone, P., Musten, L.M., et al., Short-term side effects of stimulant medication are increased in preschool children with attention-deficit/hyperactivity disorder: A double- blind placebo-controlled study. Journal of Child and Adolescent Psychopharmacology, 1998. 8(1): p. 13-25.

100. Handen, B.L., Feldman, H.M., et al., Efficacy of methylphenidate among preschool children with developmental disabilities and ADHD. Journal of the American Academy of Child and Adolescent Psychiatry, 1999. 38(7): p. 805-812.

101. Musten, L.M., Firestone, P., et al., Effects of methylphenidate on preschool children with ADHD: Cognitive and behavioral functions. Journal of the American Academy of Child and Adolescent Psychiatry, 1997. 36(10): p. 1407-1415. 
102. Swanson, J., Greenhill, L., et al., Stimulant-related reductions of growth rates in the PATS. Journal of the American Academy of Child and Adolescent Psychiatry, 2006. 45(11): p. 1304-1313.

103. Wigal, T., Greenhill, L., et al., Safety and tolerability of methylphenidate in preschool children with ADHD. Journal of the American Academy of Child and Adolescent Psychiatry, 2006. 45(11): p. 1294-1302.

104. Biederman, J., Faraone, S., et al., Psychiatric Comorbidity among Referred Juveniles with Major Depression - Fact or Artifact. Journal of the American Academy of Child and Adolescent Psychiatry, 1995. 34(5): p. 579-590.

105. Wong, I., Asherson, P., et al., Cessation of attention deficit hyperactivity disorder drugs in the young (CADDY) - a pharmacoepidemiological and qualitative study. Health Technol Assess, 2009. 13(50): p. 1-144.

106. Biederman, J., Wilens, T., et al., Psychoactive Substance Use Disorders in Adults with Attention-Deficit Hyperactivity Disorder (Adhd) - Effects of Adhd and Psychiatric Comorbidity. American Journal of Psychiatry, 1995. 152(11): p. 1652-1658.

107. Stein, M.A., Seymour, K.E., et al., Effects and side effects of Concerta methylphenidate (MPH) in children with ADHD and comorbid internalizing symptoms. Pediatric Research, 2003. 53(4): p. 555a-555a.

108. Wilens, T., Gignac, M., et al., Characteristics of adolescents and young adults with ADHD who divert or misuse their prescribed medications. Journal of the American Academy of Child and Adolescent Psychiatry, 2006. 45(4): p. 408-414.

109. Smith, B.H., Waschbusch, D.A., et al., The efficacy, safety, and practicality of treatments for adolescents with attention-deficit/hyperactivity disorder (ADHD). Clin Child Fam Psychol Rev, 2000. 3(4): p. 243-67.

110. Spencer, T., Abikoff, H.B., et al., Efficacy and safety of mixed amphetamine salts extended release (Adderall XR) in the management of oppositional defiant disorder with or without comorbid attention-deficit/hyperactivity disorder in school-aged children and adolescents: A 4-week, multicenter, randomized, double-blind, parallel- 
group, placebo-controlled, forced-dose-escalation study. Clinical Therapeutics, 2006. 28(3): p. 402-418.

111. Findling, R.L., Short, E.J., et al., Developmental aspects of psychostimulant treatment in children and adolescents with attention-deficit/hyperactivity disorder. Journal of the American Academy of Child \& Adolescent Psychiatry, 2001. 40(12): p. 1441-7.

112. Jadad, A.R., Booker, L., et al., The treatment of attention-deficit hyperactivity disorder: An annotated bibliography and critical appraisal of published systematic reviews and metaanalyses. Canadian Journal of Psychiatry-Revue Canadienne De Psychiatrie, 1999. 44(10): p. 1025-1035.

113. Chen, C.Y., Gerhard, T., et al., Determinants of Initial Pharmacological Treatment for Youths with Attention-Deficit/Hyperactivity Disorder. Journal of Child and Adolescent Psychopharmacology, 2009. 19(2): p. 187-195.

114. Coghill, D., Understudied and underrecognised: INVITED COMMENTARY ON... ATTENTION-DEFICIT HYPERACTIVITY DISORDER IN ADULTS. Adv Psychiatr Treat, 2004. 10(5): p. 338-340.

115. Castellanos, F.X., Lee, P.P., et al., Developmental trajectories of brain volume abnormalities in children and adolescents with attention-deficit/hyperactivity disorder. JAMA, 2002. 288(14): p. 1740-8.

116. Kooij, J.J.S., Burger, H., et al., Efficacy and safety of methylphenidate in 45 adults with attention-deficit/hyperactivity disorder. A randomized placebo-controlled double-blind cross-over trial. Psychological Medicine, 2004. 34(6): p. 973-982.

117. Medori, R., Ramos-Quiroga, J.A., et al., A randomized, placebo-controlled trial of three fixed dosages of prolonged-release OROS methylphenidate in adults with attention-deficit/hyperactivity disorder. Biological Psychiatry, 2008. 63(10): p. 981989.

118. Rosler, M., Fischer, R., et al., A randomised, placebo-controlled, 24-week, study of low-dose extended-release methylphenidate in adults with attentiondeficit/hyperactivity disorder (vol 259, pg 120, 2009). European Archives of Psychiatry and Clinical Neuroscience, 2009. 259(6): p. 368-368. 
119. Mattes, J.A., Boswell, L., et al., Methylphenidate effects on symptoms of attention deficit disorder in adults. . Archives of General Psychiatry, 1984. 41(11): p. 10591063.

120. Spencer, T., Biederman, J., et al., A large, double-blind, randomized clinical trial of methylphenidate in the treatment of adults with attention-deficit/hyperactivity disorder. Biological Psychiatry, 2005. 57(5): p. 456-463.

121. Spencer, T., Wilens, T., et al., A double blind crossover comparison of methylphenidate and placebo in adults with childhood onset attention deficit hyperactivity disorder. . Archives of General Psychiatry, 1995. 52(6): p. 434-443.

122. Koesters, M., Becker, T., et al., Limits of meta-analysis: methylphenidate in the treatment of adult attention-deficit hyperactivity disorder. Journal of Psychopharmacology, 2009. 23(7): p. 733-44.

123. Faraone, S.V., Spencer, T., et al., Meta-Analysis of the Efficacy of Methylphenidate for Treating Adult Attention-Deficit/Hyperactivity Disorder. Journal of Clinical Psychopharmacology, 2004. 24(1): p. 24-29.

124. Buitelaar, J.K., Ramos-Quiroga, J.A., et al., Safety and tolerability of flexible dosages of prolonged-release OROS methylphenidate in adults with attentiondeficit/hyperactivity disorder. Neuropsychiatr Dis Treat, 2009. 5: p. 457-66.

125. Horrigan, J.P. and Barnhill, L.J., Low-dose amphetamine salts and adult attentiondeficit/hyperactivity disorder. Journal of Clinical Psychiatry, 2000. 61(6): p. 414-417.

126. Spencer, T., Biederman, J., et al., Efficacy of a mixed amphetamine salts compound in adults with attention-deficit/hyperactivity disorder. Archives of General Psychiatry, 2001. 58(8): p. 775-782.

127. Weber, J. and Siddiqui, M.A.A., Lisdexamfetamine Dimesylate In Attention-Deficit Hyperactivity Disorder in Adults. CNS Drugs, 2009. 23(5): p. 419-425.

128. Ramos-Quiroga, A., Kooij, S., et al., Factors predicting treatment outcome with longacting methylphenidate in adults with attention deficit hyperactivity disorder (ADHD). ECNP Congress, 2009. 
129. Pelham, W.E., Walker, J.L., et al., Comparative effects of methylphenidate on ADD girls and ADD boys. . Journal of the American Academy of Child and Adolescent Psychiatry, 1989. 28(5): p. 773-776.

130. Barkley, R., Hyperactive girls and boys: Stimulant drug effects on mother-child interactions. Journal of Child Psychology and Psychiatry and Allied Disciplines, 1989. 30(3): p. 379-390.

131. Sonuga-Barke, E.J.S., Coghill, D., et al., Sex differences in the response of children with ADHD to once-daily formulations of methylphenidate. Journal of the American Academy of Child \& Adolescent Psychiatry, 2007. 46(6): p. 701-10.

132. Owens, E., Hinshaw, S., et al., Which treatment for whom for ADHD? Moderators of treatment response in the MTA. J Consult Clin Psychol., 2003. 74(3): p. 540-52.

133. Swanson, J., Nolan, W., et al., SNAP Rating Scale. Educational Resources in Education. 1982.

134. Adler, L., Dietrich, A., et al., Safety and tolerability of once versus twice daily atomoxetine in adults with ADHD. Annals of Clinical Psychiatry, 2006. 18(2): p. 107113.

135. Taylor, E., Schachar, R., et al., Which boys respond to stimulant medication - $a$ controlled trial of methylphenidate in boys with disruptive behavior. . Psychological Medicine, 1987. 17(1): p. 121-143.

136. Pelham, W.E., Voddehamilton, M., et al., The effects of methylphenidate on ADHD adolescents in recreational, peer group and classroom settings. . Journal of Clinical Child Psychology, 1991. 20(3): p. 293-300.

137. Munro, C.A., McCaul, M.E., et al., Sex differences in striatal dopamine release in healthy adults. Biological Psychiatry, 2006. 59(10): p. 966-74.

138. Thompson, M.J.J., Laver-Bradbury, C., et al., A small-scale randomized controlled trial of the revised new forest parenting programme for preschoolers with attention deficit hyperactivity disorder. Eur Child Adolesc Psychiatry, 2009. 18(10): p. 605-16. 
139. Sonuga-Barke, E.J.S., Daley, D., et al., Parent-based therapies for preschool attention-deficit/hyperactivity disorder: A randomized, controlled trial with a community sample. Journal of the American Academy of Child and Adolescent Psychiatry, 2001. 40(4): p. 402-408.

140. Danckaerts, M., Sonuga-Barke, E.J., et al., The quality of life of children with attention deficit/hyperactivity disorder: a systematic review. Eur Child Adolesc Psychiatry. 19(2): p. 83-105. 